\title{
Fractal Conductance Fluctuations in Electron Billiards: A Random Matrix Theory Approach
}

\author{
F. A. Pinheiro and C. H. Lewenkopf \\ Instituto de Física, Universidade do Estado do Rio de Janeiro, \\ Rua São Francisco Xavier 524, 20550-900 Rio de Janeiro, Brazil
}

Received on 4 April, 2005

\begin{abstract}
Motivated by recent experiments and theoretical works that contradict the original explanation for fractal conductance fluctuations (FCF) in electron billiards, based on the "mixed" structure of the classical phase space, we propose an alternative approach to investigate FCF using Random Matrix Theory (RMT). By means of a semiclassical estimate for value of the magnetic correlation field $B_{C}$ we conclude that most of the experiments on FCF were performed for magnetic fields around or greater than $B_{C}$. This strongly suggests that the appropriate explanation for the observed FCF should rely on the absence of long-range correlations and not on the structure of the classical phase space. This idea is supported by a numerical study of parametric variations within the framework of RMT, which validates our surmise that the observed FCF actually reflect a diffusive scenario for electronic transport.
\end{abstract}

Keywords: Fractal conductance fluctuations; Electron billiards; Random Matrix Theory

\section{INTRODUCTION}

Triggered by the pioneer theoretical prediction by Ketzmerick [1], fractal conductance fluctuations (FCF) have been experimentally observed in semiconductor billiards by several groups [2-5]. These billiards are sub-micron sized electron cavities bounded by shaped walls and connected to twodimensional electron gas reservoirs by quantum point contact leads. In such devices, where electrons are typically subjected to a "soft" potential profile [2], the classical electron dynamics is known to be "mixed", i.e., chaotic and regular regions coexist in the phase space. In open "mixed" systems the classical probability $P(t)$ that electronic trajectories stay longer in the cavity than a time $t$ (survival probability) decays as a power law $P(t) \sim t^{-\gamma}$ [7], with $\gamma \leq 2$ due to the presence of residual stable islands in the classical phase space. This is in contrast to purely chaotic systems in which $P(t)$ decays exponentially . The slow algebraic decay of the classical $P(t)$ in "mixed" systems was shown to imply, by means of semiclassical theory, that quantum fluctuation patterns of the conductance as a function of an external parameter (e.g., voltage gate or magnetic field) exhibit a fractal structure [1]. The associated fractal dimension $D_{F}$ is related to the exponent $\gamma$ according to $D_{F}=2-\gamma / 2$.

However, recent experimental results and subsequent theoretical work have revealed that the physical mechanism underlying the origin of FCF is not completely understood. From the theoretical point of view, there is evidence that both completely chaotic [8] and completely integrable billiards $[8,9]$ may exhibit fractal fluctuations [9], a result that contradicts the original prediction of Ref. [1]. On the other hand, recent experimental results [6] demonstrate that FCF are hardly affected by the change in the billiard geometry, and consequently by the classical electron trajectories. This experimental fact constitutes an unambiguous evidence that the classical electron dynamics is not as crucial as claimed originally [1] to understand FCF.

In view of this scenario, it is clear that further theoretical work is required in order to understand the physical mechanism responsible for FCF. The purpose of this paper is thus to present an alternative theoretical approach, based on Random Matrix Theory (RMT), to investigate FCF. We semiclassically estimate the magnetic correlation field and conclude that most of recent experiments [2-5] on FCF have been performed for magnetic field values beyond the magnetic correlation field. This results strongly suggests that the physical origin of the observed FCF is associated with the absence of long-range correlations related to a diffusive scenario and not on the "mixed" structure of the subjacent classical phase space. A numerical study of parametric variations within the RMT is presented to validate this hypothesis.

This paper is organized as follows. In Sec. II we present a semiclassical estimate for the magnetic correlation field $B_{C}$. In Sec. III we numerically study parametric variations within the framework of RMT in order to investigate FCF. Finally, Sec. IV is devoted to final remarks and conclusions.

\section{SEMICLASSICAL ESTIMATE FOR $X_{C}$}

Let us start by estimating the characteristic correlation field $X_{c}$, the magnetic field value beyond which the conductance values are completely uncorrelated, and comparing it to the magnetic field values involved in recent experiments. We consider a quantum dot coupled to two leads, each of them supporting $N_{c}$ perfectly transmitting channels. For chaotic systems it is well established that the conductance autocorrelation function $C(X, E)$ in the presence of an external parameter $X$ is given by [10]:

$$
C(X, E)=\frac{1}{16} \frac{1}{\left\{1+\left[X \pi /\left(X_{c} \sqrt{N_{c}}\right)\right]^{2}\right\}^{2}+(E / \Gamma)^{2}} .
$$

The correlation parameter is defined as [10]

$$
X_{C} \equiv(2 \hbar \Gamma / \alpha)^{1 / 2},
$$


where $\Gamma=\Delta N_{c} / 2 \pi$ is the energy resonance width, with $\Delta$ the mean level spacing. $\alpha$ describes the diffusive time evolution of $Q(t)$ according to [11]

$$
\left\langle Q^{2}(\tau)\right\rangle=\alpha \tau
$$

In Eq. (3) the quantity $Q(\tau)$ is defined as the derivative $Q(\tau) \equiv$ $\partial \delta S(\tau) / \partial X$ of the reduced classical action $\delta S(\tau)$

$$
\delta S(\tau)=\int_{0}^{\tau} d t H(t)
$$

where $H(t)$ is the system Hamiltonian. To estimate $\alpha$ semiclassically, let us assume that the electrons (effective mass $m^{*}$, charge $e$ ) inside the billiard in the presence of an external magnetic field $\mathbf{B}$ are described by the general Hamiltonian:

$$
H \cong \frac{p^{2}}{2 m^{*}}-\frac{e}{m^{*} c} \mathbf{p} \cdot \mathbf{A}+V(\mathbf{r})
$$

where $\mathbf{A}$ is the vector potential. If we assume, without loss of generality, that the external magnetic field is in the $z$ direction, $\mathbf{B}=B \hat{z}$, the dependence of the Hamiltonian on the magnetic field is contained in the term

$$
H_{B}=\frac{e}{2 m^{*} c} L_{z} B
$$

where $L_{z}$ is the $z$ component of the orbital angular momentum $\mathbf{L}=\mathbf{r} \times \mathbf{p}$. Inserting (6) into Eq. (4) and using the definition of $Q(t)$ one arrives at

$$
Q(t) \equiv \frac{\partial \delta S}{\partial B}=\frac{e}{2 m^{*} c} \int_{0}^{\tau} d t L_{z}(t)
$$

The second moment of $Q(t)$ is thus given by

$$
\left\langle Q^{2}(t)\right\rangle=\left(\frac{e}{2 m^{*} c}\right)^{2} C_{L}
$$

with

$$
C_{L} \equiv\left\langle\int_{0}^{\tau} d t L_{z}(t) \int_{0}^{\tau} d t^{\prime} L_{z}\left(t^{\prime}\right)\right\rangle
$$

Taking the limit $\tau \rightarrow \infty$ one obtains

$$
\lim _{\tau \rightarrow \infty} C_{L}=2 \tau \int_{0}^{\infty} d t\left\langle L_{z}(0) L_{z}(t)\right\rangle
$$

It is useful to introduce the new variables

$$
\begin{array}{r}
\tilde{r}=r / \sqrt{A}, \\
\tilde{v}=v / v_{F},
\end{array}
$$

where $v_{F}$ is the Fermi velocity and $A$ is the billiard area, we can re-scale the quantity $C_{L}$ [Eq. (10)] as

$$
C_{L}=m^{* 2} v_{F} A^{3 / 2} C_{\tilde{L}}
$$

where $C_{\tilde{L}}$ is defined analogously to Eq. (9) with $\tilde{\mathbf{L}}=\tilde{\mathbf{r}} \times \tilde{\mathbf{v}}$. Inserting relation Eq. (13) into Eq. (8) and using (10) we identify $\alpha$ as

$$
\alpha=\frac{1}{2}\left(\frac{e}{c}\right)^{2} v_{F} A^{3 / 2} C_{\tilde{L}}
$$

The final expression for the magnetic correlation field $B_{C}$ can be obtained by plugging relation (14) into the general definition (2) for the correlation parameter $X_{C}$ :

$$
B_{C}=\frac{2 \pi^{1 / 4}}{(2 \pi)^{3 / 2}} \times \frac{N^{1 / 2}}{n_{e}^{1 / 4} C_{\tilde{L}}^{1 / 2}} \times\left(\frac{\phi_{0}}{A}\right)
$$

with $\phi_{0}=h c / e$ the unit flux quantum, $n_{e}=\pi m^{* 2} v_{F}^{2} A / h^{2}$ the number of electrons inside the billiard.

We now can use the semiclassical result (15) to estimate the value of the magnetic correlation field $B_{C}$ in recent experiments on FCF [2-5]. In these experiments, one has typically $N_{c} \sim 2, n_{e} \sim 10^{2}, A \sim 1 \mu \mathrm{m}^{2}$ and $C_{\tilde{L}} \sim 10^{-1}$ so that the correlation field is of the order of $B_{C} \sim 10^{-3} \mathrm{~T}$. Some of these experiments [2-4] were performed with magnetic field values around or greater than $B_{C} \sim 10^{-3} \mathrm{~T}$, which we believe to make the interpretation of the observed conductance fluctuations as genuine FCF ambiguous. The situation is even more critical in the experiments reported on Ref. [5], in which the values of the magnetic field are far beyond $B_{C} \sim 10^{-3} \mathrm{~T}$, where the conductance values are completely uncorrelated and conductance fluctuations are not expected to occur. This encourages us to consider an alternative explanation, based on RMT, for these experimental results and which will be discussed in the following section.

\section{RMT APPROACH TO FCF: NUMERICAL RESULTS AND DISCUSSIONS}

In order to investigate FCF within the RMT, we need to generate random Hamiltonians that depend (non-periodically) on an arbitrary parameter $X, H=H(X)$, according to the Gaussian Unitary Ensemble (GUE). We shall adopt here a modified version of the method proposed in Ref. [12] to include non-periodic parametric variations in the RMT. According to this method, the $M \times M$ matrix is defined according to:

$$
[H(X)]_{i j}=\int f\left(X-X^{\prime}\right) w_{i j}\left(X^{\prime}\right) d X^{\prime},
$$

where $f(X)$ is some smooth and differentiable varying function of $X$. In the present case $w_{i j}(X)$ is a Gaussian white-noise matrix generated according to the GUE: 


$$
\begin{aligned}
\left\langle w_{i j}(X)\right\rangle & =0 \\
\left\langle w_{i j}(X) w_{k l}\left(X^{\prime}\right)\right\rangle & =v \delta_{i l} \delta_{j k} \delta\left(X-X^{\prime}\right),
\end{aligned}
$$

where $v=\left(M \Delta^{2} / 2 \pi^{2}\right)$ is the variance, $\Delta$ the mean level spacing (here set $\Delta \equiv 1$ ) and $\langle\cdots\rangle$ stands for ensemble average. Regarding the choice of $f(X)$, we shall again adopt the proposition of Ref. [12] and employ a Gaussian

$$
f(X)=\left(\frac{2}{\pi X_{0}^{2}}\right)^{1 / 4} \exp \left(-\frac{X^{2}}{X_{0}^{2}}\right)
$$

as the smooth function, although evidently this choice is not unique. Using this recipe, we are able to generate smooth matrix elements (16) over a wide range of parametric variations, much larger than the correlation value $X_{C}$.

The evaluation of the conductance $G(X, E)$ was performed using the Landauer-Bütikker formula, which relates $G(X, E)$ to the transmission coefficient $T(X, E)$ according to $G(X, E)=\left(2 e^{2} / \hbar\right) T(X, E)$. For a two-lead open device, the transmission coefficient $T(X, E)$ reads

$$
T(X, E)=\sum_{a, b=1}^{N_{c}}\left|S_{a b}(X, E)\right|^{2}
$$

where $S_{a b}(X, E)$ is the $\mathrm{S}$-matrix that can be related to system Hamiltonian $H(X)$ [13].

Figure 1 exhibits the transmission coefficient $T(X)$, calculated according the prescription described above, as a function of the the parameter $X$ for a two-lead system with $N_{c}=2$ and $M=200$ resonance poles. We consider large parametric variations, up to $100 X_{c}$, where the values of $T(X)$ are essentially uncorrelated.

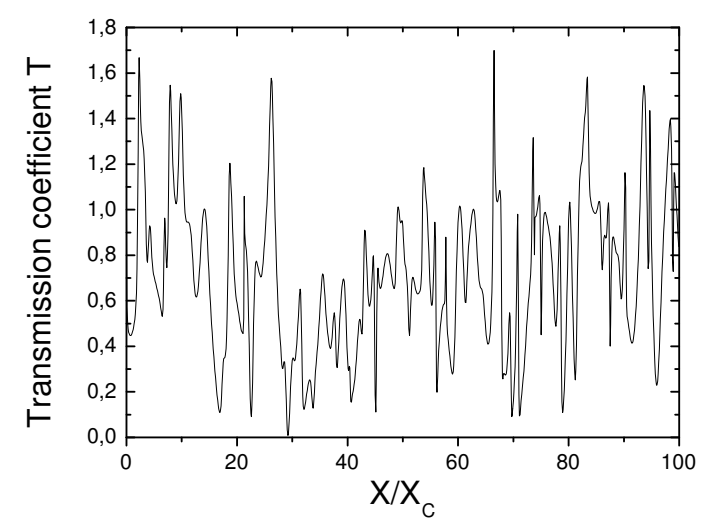

Fig. 1. Transmission coefficient as a function of the parameter $X$ for a two-lead quantum dot with $N_{c}=2$ open channels in each lead and $M=200$ resonance poles. The values of $X$ were normalized by the correlation value $X_{c}$

In order to check the numerical results, we calculate the transmission autocorrelation function $C(X, 0)$ for a two-lead quantum dot with $N_{c}=1$ and $M=2$, as exhibited in Fig. 2 . A satisfactory agreement between the numerical data and the analytical result (1) is obtained. The only input parameter is the number of open channels, $N_{c}=1$.

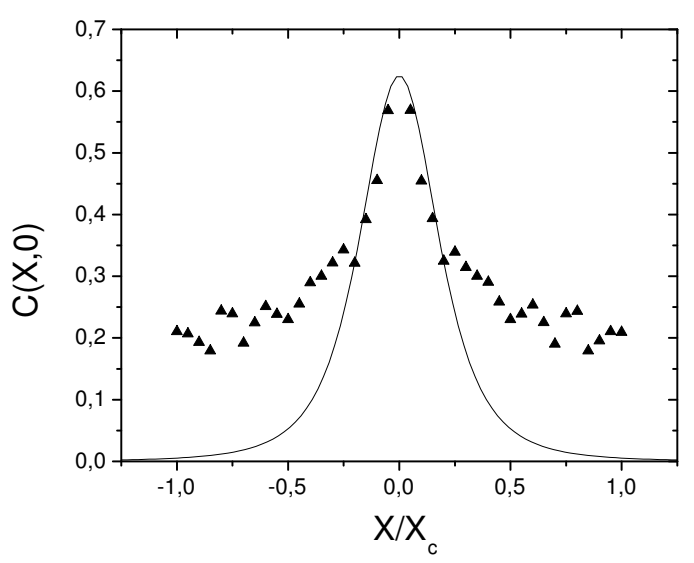

Fig. 2. Transmission autocorrelation function $C(X)$ as a function of the parameter $X$ for a two-lead quantum dot with $N_{c}=1$ and $M=2$ (filled triangles). The solid curves represents the analytical result (1).

The issue of the fractality of conductance curves is addressed by means of the calculation of their fractal dimension $D_{F}$ via the standard box-counting method [14]. The calculation of the fractal dimension of the conductance curve exhibited in Fig. 1 yields $D_{F} \cong 1.51$, a value that is very close to the fractal dimension associated with the random-walk process, $D_{F}=1.5$ [15]. This result constitutes an evidence that FCF for parametric variations much larger than the correlation value $X_{C}$, such as those calculated in Fig. 1, merely reflects a diffusive scenario for electronic transport in ballistic cavities. Curiously, the value $D_{F}=1.5$ corresponds to the theoretical maximum predicted by the original semiclassical theory for FCF [1]. More important, experimental conductance curves were shown to be fractals with corresponding fractal dimensions very close to the value $D_{F}=1.5$ [2-5], notably Ref. [5] where $D_{F}=1.44$ and Ref. [2, 4] where, for some billiards and parameters considered $D_{F} \simeq 1.5$. Remarkably, it is exactly in one of these experiments (Ref. [5]) that we argue, based on our semiclassical estimate for the magnetic correlation field $B_{C}$ presented in Sec. II, to has been performed with magnetic field values much larger than $B_{C}$. This result strongly suggests that the correct explanation for the observed FCF in some experiments should rely on the absence of long-range correlations associated with the diffusion process and not on the "mixed" structure of the subjacent classical phase space.

\section{CONCLUSIONS}

In conclusion, we have presented an alternative approach to investigate FCF based on RMT. A semiclassical estimate for the magnetic correlation field indicates that most of recent 
experiments on FCF [2-5] have been performed for magnetic field values beyond the magnetic correlation field. This results strongly suggests that the physical origin of the observed FCF is associated with the absence of long-range correlations related to a diffusive scenario and not on the "mixed" structure of the subjacent classical phase space. A numerical study of parametric variations within the framework of RMT validates this hypothesis.

\section{Acknowledgments}

This work was supported by the Brazilian founding agencies CNPq, FAPERJ and Instituto do Milênio de Nanociências.
[1] R. Ketzmerick, Phys. Rev. B 54, 10841 (1996).

[2] A. P. Micolich, R. P. Taylor, R. Newbury, J. P. Bird, R. Wirtz, C. P. Dettmann, Y. Aoyagi, and T. Sugano, J. Phys.: Condens. Matter 10, 1339 (1998).

[3] A. S. Sachrajda, R. Ketzmerick, C. Gould, Y. Feng, P. J. Kelly, A. Delage, and Z. Wasilewski, Phys. Rev. Lett. 80, 1948 (1998).

[4] A. P. Micolich, R. P. Taylor, A. G. Davies, J. P. Bird, R. Newbury, T. M. Fromhold, A. Ehlert, H. Linke, L. D. Macks, W. R. Tribe, E. H. Linfield, D. A. Ritchie, J. Cooper, Y. Aoyagi, and P. B. Wilkinson, Phys. Rev. Lett. 87, 036802 (2001)

[5] R. Crook, C. G. Smith, A. C. Graham, I. Farrer, H. E. Beere, and D. A. Ritchie, Phys. Rev. Lett. 91, 246803 (2003).

[6] A. P. Micolich, R. P. Taylor, T. P. Martin, R. Newbury, T. M. Fromhold, A. G. Davies, H. Linke, W. R. Tribe, L. D. Macks, C. G. Smith, E. H. Linfield, and D. A. Ritchie, Phys. Rev. B 70, 085302 (2004).
[7] Y. C. Lai, R. Blümel, E. Ott, and C. Grebogi, Phys. Rev. Lett. 68, 3491 (1992).

[8] E. Louis and J. A. Vergés, Phys. Rev. B 61, 13014 (2000).

[9] I. Guarneri and M. Terraneo, Phys. Rev. E 65, 015203 (2001).

[10] R. O. Vallejos and C. H. Lewenkopf, J. Phys. A: Math. Gen. 34, 2713 (2001).

[11] A. M. Ozorio de Almeida, C. H. Lewenkopf, and E. R. Mucciolo, Phys. Rev. E 58, 5693 (1998).

[12] A. Bulgac, D. D. Dang, and D. Kusnezov, Ann. Phys. 242, 1 (1995).

[13] Y. V. Fyodorov and H-J. Sommers, J. Math. Phys. (N.Y.) 38, 1918 (1997)

[14] B. B. Mandelbrot, The Fractal Geometry of Nature (Freeman, New York, 1977).

[15] S. Alexander and R. Orbach, J. Physique Lett. 43, L-625 (1982). 